\title{
Topical Issue on the Fifth International Conference on Exotic Nuclei and Atomic Masses ENAM'08
}

Published online: 14 November 2009 - (c) Società Italiana di Fisica / Springer-Verlag 2009

The International Conference on Exotic Nuclei and Atomic Masses (ENAM) has been one of the most important meetings for scientists active in the field of radioactive beams and exotic nuclei. Younger readers may not remember that it was born by merging the Atomic Mass and Fundamental Constants (AMCO) and Nuclei Far From Stability (NFFS) conference series, organized since 1956 and 1966, respectively. The first meeting under the new name of ENAM was held in Arles (France) in 1995. Since then, subsequent conferences have been organized at Bellaire, Michigan, USA (1998), Hämeenlinna, Finland (2001), and Callaway Gardens in Pine Mountain, Georgia, USA (2004). In September 2008, the fifth ENAM meeting was held in the picturesque Ryn castle located in the Polish Mazurian Lakes District. In this EPJ $A$ topical issue we offer a collection of papers presented during this last ENAM conference.

About 220 physicists from 26 countries arrived at the ENAM'08 meeting. Following the tradition, the scientific program covered a broad range of topics from atomic masses and fundamental symmetries through nuclear structure, nuclear astrophysics, synthesis and studies of heaviest elements, to reactions induced by radioactive beams and applications. As in previous years, technical subjects like production of radioactive beams as well as developments in instrumentation were also discussed. Examples of the exciting research performed in all these fields can be found in this issue.

Five days were divided into 17 plenary topical sessions. In total 106 talks (including 33 invited lectures) were delivered. The regular talks of the session were followed by a few very short oral presentations of selected posters. Overall, 150 posters were displayed throughout the conference in a separate room, and one evening was devoted to the traditional poster session.

To illustrate the variety of topics discussed at ENAM and to give some flavour of the conference, we mention a few selected examples of research highlights contained in this issue. Applications of ion traps in nuclear research keep reaching ever-widening circles, especially in precision mass spectrometry. S. Schwarz et al. report on the precision Penning trap mass measurements of rare isotopes produced by projectile fragmentation. M. Kowalska et al. present new masses of francium and radium isotopes obtained at the ISOLTRAP facility. Charge radii measured for beryllium isotopes by the isotope shift method are discussed by M. Wada et al. In-trap purification followed by post-acceleration and Coulex studies of ${ }^{61} \mathrm{Fe}$ is performed by J. Van de Walle et al. In the part devoted to fundamental interactions, a novel approach to the determination of the $V_{u d}$ CKM matrix element, employing nuclear mirror transitions, is introduced by O. Naviliat-Cuncic.

Nuclear-structure studies continue to focus on nuclei far from stability. On the neutron-rich side, the observation of beta-delayed tritons in the decay of ${ }^{11} \mathrm{Li}$ by $\mathrm{M}$. Madurga et al., as well as the studies of very heavy helium isotopes by A. Fomichev et al., are worthy of note. On the opposite side, the studies of the two-proton radioactivity are arousing interest. The first measurement of the proton-proton angular correlations from the ground-state $2 p$ radioactivity is described by K. Miernik et al. The recent application of the tracking technique to study very short-lived $2 p$ emitters is presented by I. Mukha. The current status of explorations at the upper tip of the Chart of Nuclei, including increasing evidence for elements 118 and 116 is given by Y. Oganessian. Detailed nuclear-structure studies of heavy elements follow, as shown for nobelium isotopes by R. Herzberg et al. Among new developments in reaction studies with exotic nuclei there are active targets, combining the function of a target with that of a detector, as reviewed by P. Roussel-Chomaz. A promising target system for electron scattering experiments on short-lived nuclei is described by M. Wakasugi et al.

Nuclear theorists are undertaking a comprehensive study of all nuclei based on the most accurate knowledge of the strong nuclear interaction, the most reliable theoretical approaches, and the massive use of the computer power 
available. S. Bacca et al. provide a description of halo nuclei by means of chiral low-momentum interactions, using the hyperspherical-harmonics method and coupled-cluster theory. M. Zalewski et al. discuss the structural fingerprints of tensor interaction and demonstrate that the tensor contribution to the binding energies exhibits topological features closely resembling those of the shell correction. In another interesting development, K. Yoshida studies soft vibrations in deformed neutron-rich nuclei by means of the quasiparticle random-phase approximation.

In May 2009, the decision was reached to merge the ENAM and the Radioactive Nuclear Beams meetings in order to establish the "flagship" conference representing this rapidly expanding research field. The next conference will be held in 2011 in Leuven, Belgium. While the ENAM conference series will no longer exist, the new meeting will continue to inspire and excite our growing community of scientists exploring exotic nuclei with radioactive beams.

Marek Pfützner

ENAM'08 Chair 